\title{
Intestinal obstruction in pregnancy: three case reports
}

\author{
Latha Maheswari S*, Reena Abraham, T. V. Chitra
}

\begin{abstract}
Department of Obstetrics and Gynaecology, PSG Institute of Medical Sciences \& Research, Coimbatore-641004, Tamil Nadu, India
\end{abstract}

Received: 1 August 2013

Accepted: 17 August 2013

\section{*Correspondence:}

Dr. Latha Maheswari S,

E-mail: lathamaheshwari@gmail.com

(C) 2013 Latha MS et al. This is an open-access article distributed under the terms of the Creative Commons Attribution Non-Commercial License, which permits unrestricted non-commercial use, distribution, and reproduction in any medium, provided the original work is properly cited.

\begin{abstract}
The occurrence of intestinal obstruction is uncommon in pregnancy and so it is rarely considered as a cause for acute abdominal pain. It is also a life threatening condition to the mother if proper medical intervention is delayed. In this review we present three cases of intestinal obstruction during pregnancy and puerperium. The incidence in our tertiary care center over a period of last 5 years is 1 in 3196 deliveries. These cases were diagnosed preoperatively with x-ray and sonogram. Laparotomy with definitive surgical treatment was performed. The first patient had large bowel obstruction due to sigmoid volvulus, the second patient had a stricture in ileum leading to small bowel obstruction, and the third patient had ileocecal intussusception. There were no maternal deaths but one patient had fetal loss.
\end{abstract}

Keywords: Intestinal obstruction, Pregnancy, Adhesion, Intussusception, Volvulus

\section{INTRODUCTION}

Intestinal obstruction occurs rarely in pregnancy. The incidence is not increased in pregnancy as compared to non pregnant state. When a pregnant patient presents with abdominal pain we often consider obstetric causes like preterm labour, abruption or other common surgical problems but rarely suspect surgical conditions leading to intestinal obstruction. A delay in diagnosis and treatment can lead to serious problems in mother and fetus. We present three cases with intestinal obstruction during pregnancy and puerperium and their outcome in this report.

\section{CASE REPORT 1}

A 24 year old woman G2P1L1 with 24 weeks of twin pregnancy presented to our hospital with complaints of abdominal pain, fever and constipation for 1 day. Patient gave history of undergoing surgery for gastrointestinal anomaly at birth though previous clinical records were not available. She had normal bowel habits throughout her life following the surgery. The obstetric examination findings were normal. No other mass was palpable. Initially she was treated with analgesics and laxatives. But she had no relief. So X-ray and abdominal ultrasound was taken which was suggestive of large bowel obstruction. Conservative measures were started after consulting surgical team. But she developed severe abdominal discomfort with abdominal distension with no relief of constipation over a period of four days. So surgical intervention was planned. Findings were dilated cecum, ascending, transverse, descending colon with volvulus of sigmoid colon. Volvulus was derotated, bowel decompressed and loop colostomy was done. Patient was better on first post operative day but had preterm labour on second day and delivered both fetuses. Both fetuses died in the perinatal period due complications related to extreme prematurity. Patient's condition improved and four months later underwent colostomy closure. On follow up patients bowel habits were normal.

\section{CASE REPORT 2}

This 28 year old primigravida presented at 33 weeks gestation with preterm rupture of membranes and labour pains. She delivered a live preterm baby normally but developed generalized abdominal pain, bilious vomiting 
and constipation on the second post natal day. On examination abdomen was distended with hyper peristalsis. No definite mass was palpable. There was no history of prior abdominal surgeries. Ultrasound revealed dilated bowel loops with sluggish peristalsis. CT scan showed thickened long segment \& narrowed distal jejunal loops with proximal dilatation. Though initially she was treated conservatively with nasogastric tube aspiration and IV fluids she showed no improvement and deteriorated further. Surgical intervention was planned. During surgery she was found to have a stricture in the ileum 2 feet from ileocecal junction. There was breach in small bowel $8 \mathrm{~cm}$ proximal to stricture. So resection and end to end anastomosis was carried out. No other abnormality was noted. Histopathology showed organized serosal exudate with fibrosis, stricture formation, ischemic changes with no evidence of tuberculosis. Patient had a good post operative recovery.

\section{CASE REPORT 3}

21 year old, primigravida with 21 weeks of pregnancy presented to our hospital with complaints of severe abdominal pain, vomiting and constipation for six days. She was treated in a local hospital conservatively, failing which she was referred to our hospital. On examination she had tachycardia, normal blood pressure and signs of dehydration. Her abdominal examination showed tenderness, guarding and fullness in the right hypochondrium. Uterus size corresponded to 20 weeks gestation with a live fetus. $X$ ray and ultrasound was carried out and surgery team was contacted for opinion. USG showed an air filled dilated cecum in right iliac fossa, and prominent small bowel loops with a probable diagnosis of volvulus. Emergency laparotomy was planned. The findings were ileo-caecal intussusception, with impending perforation of cecum. The omentum was attached to perforation site. The procedure carried out was limited resection of right colon. About $8 \mathrm{~cm}$ of ileum with cecum and middle $1 / 3^{\text {rd }}$ of ascending colon was resected with end to side anastomosis between ileum and ascending colon. Patient recovered completely in the post operative period and fetal condition was also stable. Histopathology report showed ileocecal intussusception with lymphoid hyperplasia of ileum.

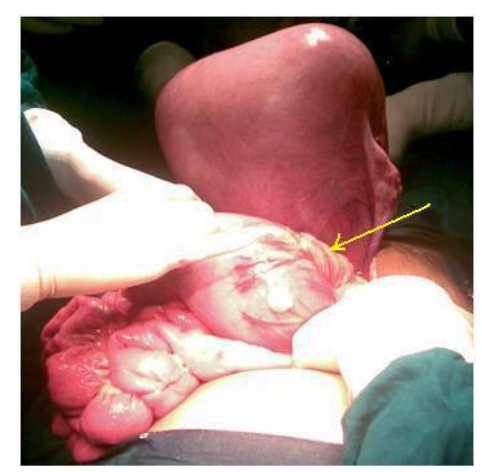

Figure 1: Per-op finding of case report 1, dilated large intestine with volvulus of sigmoid colon.

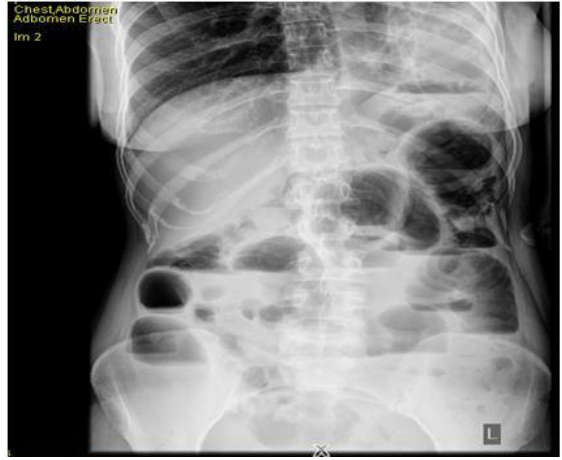

Figure 2: X-ray image of case report 2, showing multiple fluid levels with gas above; 'step ladder pattern', suggestive of bowel obstruction.

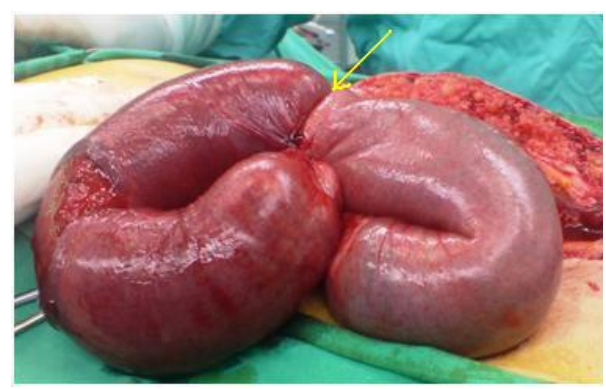

Figure 3: Per-op finding of case report 2, showing ileal structure.

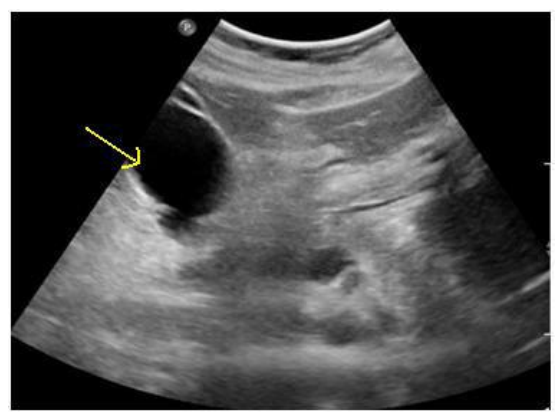

Figure 4: Ultrasound image of case report 3, showing dilated caecum.

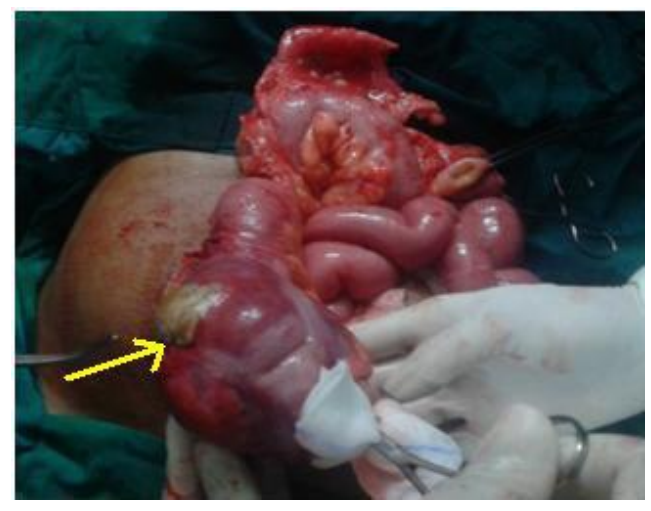

Figure 5: Per-op finding of case report 3, showing ileo-caecal intussusception, with impending perforation of caecum. 


\section{DISCUSSION}

Intestinal obstruction in pregnancy is a rare condition. Reported incidence varies between 1 in 1500 to 66,431 pregnancies. ${ }^{1}$ Although it is uncommon, intestinal obstruction in pregnancy carries significant maternal mortality of $6-20 \%^{2,3,4}$ and fetal mortality of $20-26 \%$. $^{1,3}$

Intestinal obstruction is generally classified as dynamic type and adynamic type. In dynamic type peristalsis works against a mechanical obstruction (e.g. adhesions/stricture) and in adynamic type there is no mechanical obstruction and peristalsis is absent or inadequate (e.g. paralytic ileus). The commonest cause of intestinal obstruction in pregnancy is adhesions $60 \%$. Other causes include volvulus $(25 \%)$, intussusception (5\%), stricture, hernia, carcinoma and diverticulitis/ diverticulosis., ${ }^{3,5-9}$

Dynamic intestinal obstruction is classified into small bowel obstruction-high /low and large bowel obstruction. The nature of presentation will be influenced by whether the obstruction is complete or incomplete. The cardinal clinical features of acute obstruction are abdominal pain, distension, vomiting, and absolute constipation. In our review although all our patients presented with clinical picture of dynamic bowel obstruction they had three different causes. Initially the diagnosis was difficult as symptoms like nausea, vomiting and constipation are also seen in normal pregnancy. All our three patients had similar complaints of vomiting, absolute constipation and severe abdominal discomfort not responding to conservative measures. These additional clinical features lead us to suspect a serious surgical problem necessitating a surgeons opinion. Abdominal $\mathrm{x}$-ray in pregnancy to suspect bowel obstruction was performed initially. It is an easy and simple method with a small acceptable risk (less than 5rads). Ultrasound was also used as it is another useful and safe investigation which is reliable in most of the cases. CT scan was also performed in the post natal patient and it was more informative. Though we tried initially to manage conservatively, all three patients failed to respond and needed surgical intervention. The first patient had volvulus with history of prior treatment of gastrointestinal anomaly at birth, second had ischemic ileal stricture in early post natal period and third patient had ileocolic intussusception. Except the first case other two cases had no prior abdominal surgeries. All patients underwent surgical intervention with good post operative recovery except for the first patient who had a miscarriage on second postoperative day.

\section{CONCLUSION}

Since intestinal obstruction is uncommon in pregnancy, there is possibility of delay in diagnosis and proper management. There is a hesitation to do radiological investigation in view of fetal radiation exposure and a tendency to prolong conservative management to avoid surgery during pregnancy as compared to non pregnant state. Early diagnosis and timely surgical intervention should be performed to reduce the risk of maternal and fetal mortality.

\section{REFERENCES}

1. Perdue PW, Johnson HW, Jr., Stafford PW. Intestinal obstruction complicating pregnancy. Am J Surg. 1992;164(4):384-388.

2. Coleman MT, Trianfo VA, Rund DA. Nonobstetric emergencies in pregnancy: trauma and surgical conditions. Am J Obstet Gynecol, 1997, 177;497502.

3. Connolly M M, UntiJA, Nora PF: Bowel obstruction in pregnancy. Surg Clinical North Am 1995;75:101.

4. Chiedozi LC, Ajabor LN, Iweze FI. Small intestinal obstruction in pregnancy and puerperium. Saudi J Gastroenterol 1999;5:134-9.

5. Redlich A, Rickes S, Costa SD. Small bowel obstruction in Pregnancy. Arch Gynecol Obstet. 2007;271:381.

6. Wenetick LH, Rosehen FP, Dunn JM. Volvulus of the small bowel complicating pregnancy. J Reprod Med. 1973;14:82-83.

7. Sherer DM, Abulafia O. Post cesarean small bowel volvulus case report and review of the literature. Gynecol Obstet Invest 2001:51;69-72.

8. Hill SM, Symmonds RE. Small bowel obstruction in pregnancy: A review and report of four cases. Obstet Gynecol. 1977;49:170-173.

9. Sherer D, Frager D, Eliakim R. An unusual case of diverticulitis complicating pregnancy at 33 weeks' gestation. Am J Perinatol. 2001;18:107-11.

DOI: $10.5455 / 2320-1770 . i j r \operatorname{cog} 20130957$

Cite this article as: Latha MS, Abraham R, Chitra

TV. Intestinal obstruction in pregnancy: three case reports. Int J Reprod Contracept Obstet Gynecol 2013;2:491-3. 